\section{Embedded Iterative Semi-Blind Channel Estimation for Three-Stage-Concatenated MIMO-Aided QAM Turbo-Transceivers}

\author{
Peichang Zhang, Sheng Chen, Fellow, IEEE, and Lajos \\ Hanzo, Fellow, IEEE
}

\begin{abstract}
The lack of accurate and efficient channel estimation (CE) for the multi-input multi-output (MIMO) channel state information (CSI) has long been the stumbling-block of nearMIMO-capacity operation. We propose a semi-blind joint $\mathrm{CE}$ and three-stage iterative detection/decoding scheme for near-capacity MIMO systems. The main novelty is that our decision-directed (DD) CE exploits the a posteriori information produced by the MIMO soft-demapper within the inner turbo loop for selecting a 'just-sufficient-number' of high-quality detected soft bit blocks or symbols for DD channel estimation (DDCE), which significantly improves the accuracy and efficiency of DDCE. Moreover, our DDCE is naturally embedded into the iterative three-stage detection/decoding process, without imposing an additional external iterative loop between the DDCE and the three-stage turbo detector/decoder. Hence, the computational complexity of our joint $\mathrm{CE}$ and three-stage turbo detector/decoder remains similar to that of the three-stage turbo detection/decoding scheme associated with the perfect CSI. Most significantly, the mean square error of our DD channel estimator approaches the Cramér-Rao lower bound associated with the optimal training based $\mathrm{CE}$, while the bit error rate of our semi-blind scheme is capable of achieving the optimal maximum-likelihood performance bound associated with the perfect CSI.
\end{abstract}

Index Terms-Multi-input multi-output, near-capacity, joint channel estimation and three-stage turbo detection/decoding, Cramér-Rao lower bound

\section{INTRODUCTION}

Under idealised conditions coherent multiple-input multiple-output (MIMO) systems are capable of achieving substantial diversity and/or multiplexing gains. The challenge is however the acquisition of accurate MIMO channel state information (CSI) without imposing an excessive pilotoverhead, which would erode the system's throughput too much, and without resulting in a potentially excessive channel estimation (CE) complexity. The current state-of-the-arts [1]-[16] typically combine the decision-directed (DD) CE (DDCE) with powerful iterative detection/decoding schemes to form semi-blind joint $\mathrm{CE}$ and turbo detection/decoding, where only a small number of training symbols is employed for generating an initial least squares channel estimate (LSCE). The turbo detection/decoding operation then commences with the initial LSCE. After the convergence of the turbo detector and decoder, the detected data are fed into the DDCE for the CE update. The DDCE and the turbo

Copyright (c) 2013 IEEE. Personal use of this material is permitted. However, permission to use this material for any other purposes must be obtained from the IEEE by sending a request to pubs-permissions@ieee.org.

The authors are with Electronics and Computer Science, University of Southampton, Southampton SO17 1BJ, UK. E-mails: \{pz3g09, sqc, lh\}@ecs.soton.ac.uk.

The financial supports of RC-UK under the auspices of the India-UK Advanced Technology Centre, as well as of EU Concerto project and that of the European Research Council's Advanced Fellow Grant are gratefully acknowledged. detector/decoder iterate a number of times until the channel estimate converges. The most effective schemes [10]-[13], [15], [16] employ soft-decision aided channel estimators, which are more robust against error propagation than the hard-decision aided CE schemes. Consequently, these joint soft-decision based CE and turbo detection/decoding schemes are capable of achieving better overall system-performance than their hard-decision based counterparts.

All these existing joint $\mathrm{CE}$ and turbo detection/decoding structures have a number of limitations. Firstly, an extra iterative loop between the $\mathrm{CE}$ and the turbo detector/decoder is introduced, which considerably increases the complexity imposed. Secondly, existing schemes use the entire frame of the detected soft or hard bits for CE, and the complexity of the associated DD LSCE may become unacceptably high. This is because the number of bits in a single interleaved frame of a turbo code is very large, and typically thousands of bits are contained in a turbo coded frame. Thirdly and most importantly, at the low signal-to-noise ratios (SNRs) practically $50 \%$ of the detected bits are erroneous and hence the error propagation may still be serious even for soft-decision aided CE schemes. Hence error-propagation would severely degrade the achievable performance. Therefore, all these existing schemes fail to approach the optimal maximum-likelihood (ML) turbo detection performance bound associated with the perfect CSI. In other words, even the best known joint CE and turbo detection/decoding schemes are incapable of attaining the optimal performance bound of the idealised ML turbo detector/decoder associated with the perfect CSI. Hence it would appear that it is necessary to implant a substantial pilotoverhead, which dramatically erodes the system's throughput.

The objective of our current study is to demonstrate that the optimum MIMO performance may nonetheless be approached with the aid of a very modest training overhead and without a significant increase in computational complexity. Specifically, we develop a novel joint $\mathrm{CE}$ and three-stage iterative detection/decoding structure ${ }^{1}$ for near-capacity MIMO systems [19]. Our original contribution is twofold.

1) Firstly, we propose a block-of-bits selection based softdecision aided CE (BBSB-SCE) scheme, which selects the high-quality or more reliable detected symbols or blocks of bits based on the a posteriori information produced by the MIMO soft-demapper within the original inner turbo loop of the unity-rate-code (URC) decoder and MIMO detector advocated. Since our BBSB-SCE scheme only utilises a 'just-sufficient-number' of the high-quality detected symbols for $\mathrm{CE}$, in contrast to the existing state-of-the-art solutions, it does not suffer from the usual performance degradation imposed by erroneous decisions. Furthermore, this measure dramatically reduces the complexity of the DD LSCE.

\footnotetext{
${ }^{1}$ The low-complexity memory-1 unity-rate-code adopted has an innite impulse response, which allows the system to spread the extrinsic information benecially across the iterative decoder components without increasing its delay. Therefore, the EXtrinsic Information Transfer (EXIT) curve is capable of reaching the $(1.0,1.0)$ point of perfect convergence in the EXIT charts, which is a necessary condition for near-capacity operation and for achieving a vanishingly low bit error rate [17]-[20].
} 
2) Secondly, our CE is naturally embedded in the original three-stage turbo detection/decoding process, and no extra iterative loop is required between the $\mathrm{CE}$ and the three-stage MIMO detector/decoder. Hence, the complexity of our joint BBSB-SCE and three-stage turbo detector/decoder remains similar to that of the idealised three-stage turbo receiver relying on the perfect CSI. We will show that our scheme is capable of attaining the optimal ML performance bound of the idealised threestage turbo detector/decoder associated with the perfect CSI, despite using the same number of turbo iterations as the latter.

\section{Joint CE And Three-Stage Turbo Receiver}

We consider a MIMO system relying on $N_{T}$ transmit antennas and $N_{R}$ receive antennas for communication in a frequency-flat Rayleigh fading environment. High-order quadrature amplitude modulation (QAM) [21] is used.

\section{A. MIMO System Model}

The transmitter consists of a two-stage serial-concatenated outer recursive systematic code (RSC) encoder combined with an inner URC encoder, followed by the MIMO-aided $L$-QAM modulator. This scheme is capable of achieving a near-capacity performance under idealised conditions [19]. The number of bits per $L$-QAM symbol (BPS) is given by BPS $=\log _{2}(L)$, and let $i$ denote the symbol index. The MIMO system model is expressed as

$$
\boldsymbol{y}(i)=\boldsymbol{H} \boldsymbol{s}(i)+\boldsymbol{v}(i),
$$

where $\boldsymbol{H} \in \mathbb{C}^{N_{R} \times N_{T}}$ is the MIMO channel matrix whose elements obey the complex-valued zero-mean Gaussian distribution $\mathcal{C N}(0,1)$ with a variance of $\frac{1}{2}$ per dimension, $s(i) \in \mathbb{C}^{N_{T}}$ is the transmitted $L$-QAM symbol vector, $\boldsymbol{y}(i) \in \mathbb{C}^{N_{R}}$ is the received signal vector, and $\boldsymbol{v}(i) \in \mathbb{C}^{N_{R}}$ is the noise vector whose elements obey $\mathcal{C N}\left(0, N_{\mathrm{o}}\right)$ with a variance of $\frac{N_{\mathrm{o}}}{2}$ per dimension. The system's SNR is defined as $\mathrm{SNR}=E_{\mathrm{s}} / N_{\mathrm{o}}$, where $E_{\mathrm{s}}$ is the average symbol energy.

Let us define the number of bits per block (BPB) as $\mathrm{BPB}=N_{T}$. BPS. At the receiver, upon obtaining the a priori log-likelihood ratios (LLRs) $\left\{L_{a}\left(u_{k}\right)\right\}_{k=1}^{\mathrm{BPB}}$ from the channel decoder, where $\left\{u_{k}\right\}_{k=1}^{\mathrm{BPB}}$ indicate the corresponding bits that are mapped to the symbol vector $s(i)$, the a posteriori LLRs produced by the ML MIMO soft-demapper ${ }^{2}$ are expressed as [22]

$$
L_{p}\left(u_{k}\right)=L_{p}(k)=\ln \frac{\sum_{\boldsymbol{s}^{n} \in\left\{\boldsymbol{s}_{u_{k}=1}\right\}} \exp \left(p_{n}\right)}{\sum_{\boldsymbol{s}^{n} \in\left\{\boldsymbol{s}_{u_{k}=0}\right\}} \exp \left(p_{n}\right)},
$$

where $\left\{\boldsymbol{s}_{u_{k}=1}\right\}$ and $\left\{\boldsymbol{s}_{u_{k}=0}\right\}$ represent the $L$-QAM symbol vector sets with the corresponding bit $u_{k}=1$ and $u_{k}=0$,

\footnotetext{
${ }^{2}$ For large MIMO systems, we may opt for using reduced-complexity nearoptimum detection schemes, e.g. the K-best sphere detector [23], [24], to avoid the exponentially increasing complexity imposed by the ML detector.
}

respectively. The probability metrics $\left\{p_{n}\right\}_{n=1}^{L^{N_{T}}}$ for the possible $L$-QAM symbol vectors $\left\{\boldsymbol{s}^{n}\right\}_{n=1}^{L^{N_{T}}}$ are given as

$$
p_{n}=-\frac{\left\|\boldsymbol{y}(i)-\boldsymbol{H} \boldsymbol{s}^{n}\right\|^{2}}{N_{0}}+\sum_{k=1}^{\mathrm{BPB}} \tilde{u}_{k} L_{a}\left(u_{k}\right),
$$

where $\left\{\tilde{u}_{k}\right\}_{k=1}^{\mathrm{BPB}}$ indicate the corresponding bits that map to the specific symbol vector $s^{n}$.

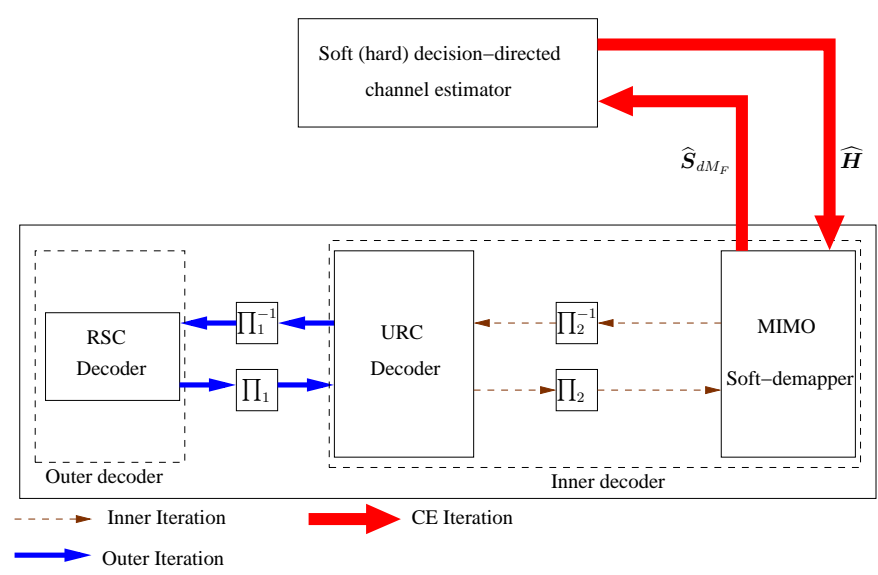

Fig. 1: Conventional joint CE and three-stage turbo detector/decoder, where $\widehat{\boldsymbol{S}}_{\mathrm{d} M_{F}}$ denotes all the soft or hard detected symbol blocks corresponding to the received data frame. Note that all the detected bits are used by the decision-directed channel estimator. In order to benefit from the full error correction capability of the three-stage turbo detection/decoding, the updating of the DDCE takes place after the convergence of the outer turbo loop.

\section{B. State-of-the-arts}

The state-of-the-arts [1]-[16] can be represented by the conventional joint $\mathrm{CE}$ and three-stage turbo detector/decoder structure $^{3}$ depicted in Fig. 1. For initiating the joint CE and three-stage turbo detection/decoding process an initial training based channel estimate is required. Let us assume that the number of available training blocks is $M_{T}$ and the initial training data are arranged as $\boldsymbol{Y}_{t M_{T}}=\left[\boldsymbol{y}(1) \boldsymbol{y}(2) \cdots \boldsymbol{y}\left(M_{T}\right)\right]$ and $\boldsymbol{S}_{t M_{T}}=\left[\boldsymbol{s}(1) \boldsymbol{s}(2) \cdots \boldsymbol{s}\left(M_{T}\right)\right]$. Then the LSCE of the MIMO channel matrix $\boldsymbol{H}$ is given by

$$
\widehat{\boldsymbol{H}}_{L S C E}=\boldsymbol{Y}_{t M_{T}} \boldsymbol{S}_{t M_{T}}^{\mathrm{H}}\left(\boldsymbol{S}_{t M_{T}} \boldsymbol{S}_{t M_{T}}^{\mathrm{H}}\right)^{-1},
$$

where $(\cdot)^{\mathrm{H}}$ denotes the conjugate transpose operator. To maintain a high system throughput, only a small number of training blocks is used. To ensure a full rank for $\boldsymbol{S}_{t M_{T}} \boldsymbol{S}_{t M_{T}}^{\mathrm{H}}$, it is necessary to choose $M_{T} \geq N_{T}$. Therefore, $N_{T}$ is a lower bound for the number of initial training blocks. With $M_{T}$ chosen to be close to its lower bound $N_{T}$, the accuracy of the LSCE (4) is poor, hence the achievable bit error ratio (BER) based on this initial LSCE is also poor. However, the three-stage turbo detector/decoder is capable of improving the reliability of the detected bits for assisting the soft decisiondirected $\mathrm{CE}$, which then provides a more accurate channel

\footnotetext{
${ }^{3}$ Most of these schemes were originally designed for the two-stage turbo detector/decoder structure, but they can be readily extended to the three-stage turbo detector/decoder structure discussed here.
} 
estimate. This iterative process results in an increasingly more reliable turbo detector/decoder output. Observe from Fig. 1 that since all the detected bits are used by the channel estimator, the DDCE update operation takes place after the convergence of the three-stage turbo detection/decoding, in order to fully exploit the error correction capability of the three-stage turbo detector/decoder. This introduces the additional CE loop shown in Fig. 1.

Let $M_{F}$ be the length of the MIMO observation data sequence, which is arranged as

$$
\boldsymbol{Y}_{\mathrm{d} M_{F}}=\left[\boldsymbol{y}(1) \boldsymbol{y}(2) \cdots \boldsymbol{y}\left(M_{F}\right)\right] .
$$

Let $C_{\mathrm{RSC}}, C_{\mathrm{URC}}$ and $C_{\mathrm{ML}}$ denote the computational complexity of the RSC decoder, the URC decoder, and the ML soft-demapper, respectively. Assume that given the CSI, the two-stage inner turbo loop requires $I_{\text {in }}$ iterations, while the outer turbo loop requires $I_{\text {out }}$ iterations. Then, given the CSI, the computational complexity of the three-stage turbo receiver can be formulated as

$$
C_{\text {ideal }}=I_{\text {out }}\left(C_{\mathrm{RSC}}+I_{\mathrm{in}}\left(C_{\mathrm{ML}}+C_{\mathrm{URC}}\right)\right) .
$$

The CE loop of Fig. 1 requires $I_{\mathrm{ce}}$ iterations to converge and the computational complexity of its $\mathrm{CE}$ is on the order of $M_{F}^{3}$, or $\mathcal{O}\left(M_{F}^{3}\right)$, which is extremely high, considering the fact that $M_{F}$ is typically in the thousands. Thus, the overall computational complexity of the conventional joint $\mathrm{CE}$ and three-stage turbo receiver can be expressed as

$$
C_{\text {con }}=I_{\text {ce }} \cdot \mathcal{O}\left(M_{F}^{3}\right)+I_{\text {ce }} \cdot C_{\text {ideal }}
$$

which is significantly higher than $C_{\text {ideal }}$. More importantly, the frame of the detected bits may contain a large percentage of erroneous decisions, particularly at the low SNRs, which will degrade even the soft decision-directed channel estimator that utilize all the soft detected symbol blocks $\widehat{\boldsymbol{S}}_{\mathrm{d} M_{F}}$ corresponding to the received data frame $\boldsymbol{Y}_{\mathrm{d} M_{F}}$. Therefore the existing conventional joint $\mathrm{CE}$ and three-stage turbo receivers fail to approach the optimal BER performance bound of the idealised three-stage turbo ML-detector/decoder associated with the perfect CSI [10]-[12].

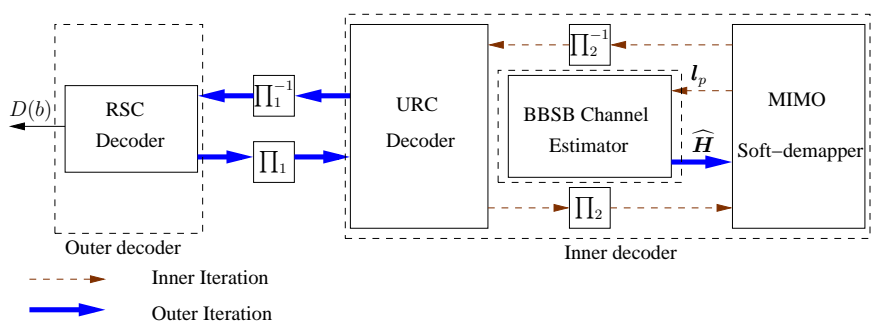

Fig. 2: Proposed joint BBSB-SCE and three-stage turbo detector/decoder. Because only reliable detected bit blocks are utilised in the decision-directed channel estimation, there is no need to wait for the convergence of the three-stage turbo detector/decoder, and the updating of the DDCE can take place concurrently with the outer turbo iteration.

\section{Joint BBSB-SCE and Three-Stage Turbo Receiver}

The novel structure of our proposed BBSB-SCE and threestage turbo detector/decoder is depicted in Fig. 2. Note that there is no additional iterative loop involving the CE and the three-stage turbo detector/decoder. In other words, our soft decision aided CE is embedded in the original outer loop of the three-stage turbo structure, and the CE update occurs concurrently with the original outer turbo decoding iteration. Moreover, our CE does not use the entire frame of the detected bits. Rather, it only selects the high-quality or reliable decisions. Specifically, the a posteriori information (2) output by the MIMO soft-demapper provides the confidence levels of binary 1s and 0s [19]. Therefore, based on this confidence level, we can select the reliable decisions from the MIMO soft-demapper's output sequence for CE. Removing most of the erroneous decisions from the CE leads to a much more accurate channel estimate, which in turn enhances the performance of the three-stage turbo detection/decoding process. Consequently, our joint BBSB-SCE and three-stage turbo detector/decoder is capable of attaining the performance bound of the idealised three-stage turbo ML-detector/decoder associated with the perfect CSI, as will be confirmed in our simulation study. As a further benefit of only selecting reliable decisions, the complexity of our soft-decision aided LSCE is dramatically lower than $\mathcal{O}\left(M_{F}^{3}\right)$. Let us now detail our scheme further.

Step 1) Set the outer turbo iteration index to $t=0$ and the initial channel estimate to $\widehat{\boldsymbol{H}}^{(t)}=\widehat{\boldsymbol{H}}_{L S C E}$.

Step 2) Given $\widehat{\boldsymbol{H}}^{(t)}$, perform the ML soft-demapping for the observation data $\boldsymbol{Y}_{\mathrm{d} M_{F}}$ of (5). The MIMO soft-demapper exchanges its soft information with the URC inner decoder for $I_{\text {in }}$ iterations, yielding the $I_{\text {in }}$ vectors of the a posteriori information as defined in (2), which can be arranged as the following a posteriori information matrix

$$
\boldsymbol{L}_{p}=\left[\boldsymbol{l}_{p}^{1} \boldsymbol{l}_{p}^{2} \cdots \boldsymbol{l}_{p}^{I_{\mathrm{in}}}\right]^{\mathrm{T}} \in \mathbb{C}^{I_{\mathrm{in}} \times L_{F}},
$$

where $(\cdot)^{\mathrm{T}}$ denotes the transpose operator, $L_{F}=\mathrm{BPB}$. $M_{F}$ is the total number of bits in a frame, $l_{p}^{i}=$ $\left[L_{p}^{i}(1) L_{p}^{i}(2) \cdots L_{p}^{i}\left(L_{F}\right)\right]^{\mathrm{T}} \in \mathbb{C}^{L_{F}}$ for $1 \leq i \leq I_{\text {in }}$ is the a posteriori information vector obtained during the $i$ th inner iteration. The $n$th column of $\boldsymbol{L}_{p}$ contains the $I_{\text {in }}$ soft decisions $\left\{L_{p}^{1}(n), L_{p}^{2}(n), \cdots, L_{p}^{I_{\text {in }}}(n)\right\}$ for the $n$th information bit obtained in the $I_{\text {in }}$ inner decoder iterations, which we exploit to judge whether the $n$th detected bit is reliable or not. Specifically, the $n$th detected bit is judged to be high quality when either of the following two criteria is met:

Criterion 1: If the soft decisions in the $n$th column of $\boldsymbol{L}_{p}$ share similar values, these soft decisions may result in a stable and reliable bit decision, which is hence considered to be correct. Specifically, the criterion for the $n$th detected bit to be judged as a correct one is

$$
\frac{\left|L_{p}^{1}(n)-L_{p}^{2}(n)\right|+\cdots+\left|L_{p}^{I_{\text {in }}-1}(n)-L_{p}^{I_{\text {in }}}(n)\right|}{|\mu|} \in\left(0, T_{h}\right),
$$

where $\mu$ is the mean of the soft decisions in the $n$th column of $\boldsymbol{L}_{p}$, and $T_{h}$ denotes the pre-defined block-of-bits selection threshold. 
Criterion 2: If the absolute values of the soft decisions in the $n$th column of $\boldsymbol{L}_{p}$ are in monotonically ascending order and these soft decisions share the same sign, namely,

$$
\begin{gathered}
\left|L_{p}^{1}(n)\right|<\left|L_{p}^{2}(n)\right|<\cdots<\left|L_{p}^{I_{\text {in }}}(n)\right| \text { and } \\
\operatorname{sign}\left\{L_{p}^{1}(n)\right\}=\operatorname{sign}\left\{L_{p}^{2}(n)\right\}=\cdots=\operatorname{sign}\left\{L_{p}^{I_{\text {in }}}(n)\right\},
\end{gathered}
$$

the $n$th detected bit may be regarded as a correct one.

By checking through the columns of $\boldsymbol{L}_{p}$, only high-confidence decision blocks are selected and the corresponding symbol block indices can be determined by a sliding-window method using a window-size of BPB bits. More explicitly, only when the BPB consecutive detected bits of a block are all regarded as correct, the corresponding symbol vector is selected for CE. This process yields an integer-index vector, denoted as $\boldsymbol{x}^{t}=\left[x^{t}(1) x^{t}(2) \cdots x^{t}\left(M_{s}^{t}\right)\right]^{\mathrm{T}}$ in which $x^{t}(i)$ is the position or index of the $i$ th selected symbol vector in the transmitted symbol vector sequence. The number of the selected symbol vectors $M_{s}^{t}$ varying within $\left\{1,2, \cdots, M_{\text {sel }}\right\}$, where $M_{\text {sel }} \ll M_{F}$ is the maximum number of blocks imposed for CE. Specifically, whenever the number of selected reliable symbol vectors $M_{s}^{t}$ reaches the limit $M_{\text {sel }}$, the sliding-window process ends; otherwise, the sliding-window process examines all the possible bit blocks and outputs the $M_{s}^{t}$ selected symbol vectors. Thus, $M_{s}^{t}$ varies at each outer turbo iteration $t$, and $M_{s}^{t} \leq M_{\text {sel }}$. By using this index vector, the corresponding observation data can be selected from (5), and re-arranged as

$$
\boldsymbol{Y}_{\mathrm{sel}}^{(t)}=\left[\boldsymbol{y}\left(x^{t}(1)\right) \boldsymbol{y}\left(x^{t}(2)\right) \cdots \boldsymbol{y}\left(x^{t}\left(M_{s}^{t}\right)\right)\right] .
$$

Step 3) Based on the selected high-confidence detected blocks of bits having the symbol vector indices $\boldsymbol{x}^{t}$, generate the softestimate of each symbol element as [13]

$$
\begin{aligned}
\widehat{s}^{m}\left(x^{t}(n)\right) & =\sum_{l=1}^{L} s^{l} \operatorname{Pr}\left\{s^{m}\left(x^{t}(n)\right)=s^{l}\right\} \\
& =\sum_{l=1}^{L} s^{l} \cdot \frac{\exp \left(\sum_{j=1}^{\mathrm{BPS}} \widetilde{u}_{j} L_{a}\left(u_{j}\right)\right)}{\prod_{j=1}^{\mathrm{BPS}}\left(1+\exp \left(L_{a}\left(u_{j}\right)\right)\right)},
\end{aligned}
$$

for $1 \leq n \leq M_{s}^{t}$, where $\left\{s^{l}\right\}_{l=1}^{L}$ denotes the $L$-QAM symbol set, $m \in\left\{1,2, \cdots, N_{T}\right\}$ indicates the symbol index in the soft-estimated symbol vector $\widehat{\boldsymbol{s}}\left(x^{t}(n)\right)$, and $\left\{\widetilde{u}_{j}\right\}_{j=1}^{\text {BPS }}$ represents the bit mapping corresponding to $\left\{s^{l}\right\}_{l=1}^{L}$. By arranging the soft-estimated symbol vectors as

$$
\widehat{\boldsymbol{S}}_{\mathrm{sel}}^{(t)}=\left[\widehat{\boldsymbol{s}}\left(x^{t}(1)\right) \widehat{\boldsymbol{s}}\left(x^{t}(2)\right) \cdots \widehat{\boldsymbol{s}}\left(x^{t}\left(M_{s}^{t}\right)\right)\right],
$$

as well as defining $\boldsymbol{Y}_{\mathrm{t}+\mathrm{sel}}^{(t)}=\left[\begin{array}{ll}\boldsymbol{Y}_{t M_{T}} & \boldsymbol{Y}_{\text {sel }}^{(t)}\end{array}\right]$ and $\widehat{\boldsymbol{S}}_{\mathrm{t}+\mathrm{sel}}^{(t)}=$ $\left[\begin{array}{ll}\boldsymbol{S}_{t M_{T}} & \widehat{\boldsymbol{S}}_{\mathrm{sel}}^{(t)}\end{array}\right]$, the resulting decision-directed LSCE is given by

$$
\widehat{\boldsymbol{H}}^{(t+1)}=\boldsymbol{Y}_{\mathrm{t}+\mathrm{sel}}^{(t)}\left(\widehat{\boldsymbol{S}}_{\mathrm{t}+\mathrm{sel}}^{(t)}\right)^{\mathrm{H}}\left(\widehat{\boldsymbol{S}}_{\mathrm{t}+\mathrm{sel}}^{(t)}\left(\widehat{\boldsymbol{S}}_{\mathrm{t}+\mathrm{sel}}^{(t)}\right)^{\mathrm{H}}\right)^{-1} .
$$

This update occurs as the soft information is exchanged between the two-stage inner decoder and the outer RSC decoder, as indicated in Fig. 2.

Step 4) Set $t=t+1$. If $t<I_{\text {out }}$, repeat Steps 2) and 3); otherwise, stop.
The computational complexity of our $\mathrm{CE}$ is upper bounded by $\mathcal{O}\left(M_{\text {sel }}^{3}\right)$ which is much smaller than $\mathcal{O}\left(M_{F}^{3}\right)$. For example, considering a reasonable case of $M_{F}=1000$ and $M_{\text {sel }}=100$, the complexity of our CE is more than 1000 times smaller than that of the conventional scheme. The total complexity of our proposed scheme satisfies

$$
C_{\text {pro }} \leq I_{\text {out }} \cdot \mathcal{O}\left(M_{\text {sel }}^{3}\right)+C_{\text {ideal }}
$$

Since $I_{\text {out }} \cdot \mathcal{O}\left(M_{\text {sel }}^{3}\right) \ll C_{\text {ideal }}$, we have $C_{\text {pro }} \approx C_{\text {ideal }}$.

Remark 1. We now elaborate on the two criteria used for selecting high-quality bits. The idea behind Criterion 1 is that if the decisions for the $n$th bit are relatively similar during the inner turbo iterations, the $n$th bit decision may be regarded as reliable. This makes sense because following a number of outer iterations, a stable state may be reached by the turbo decoder and hence the stable decisions of the inner decoder are likely to be the correct ones. Our experience suggests that most of the chosen bit blocks or symbols are selected according to Criterion 1.

As for Criterion 2, we note that if the absolute values of the decisions for a specific bit are in monotonically ascending order and these decisions share the same polarity, the corresponding bit decision is likely to be correct. This makes sense because the correct decisions may experience iteration gain and this will lead to increasing absolute values of the softdecisions as the number of inner iterations increases. This type of reliable decisions could be missed according to Criterion 1 and hence Criterion 2 allows us to select these high-quality decisions when they do occur.

An important point to note is that our scheme fully exploit the information provided by the entire inner turbo iterative process, as manifested in the $n$th column of the a posteriori information matrix $\boldsymbol{L}_{p}$ in (8). Therefore, it is capable of making a high-confidence decision regarding whether the $n$th detected bit is reliable or not.

Remark 2. For binary phase shift keying (BPSK) signalling, the work of Abe and Matsumoto [25] also selects reliable decisions for DDCE. Specifically, a soft symbol estimate is directly obtained from the bit's LLR [25], owing to the one bit per symbol nature of BPSK. The magnitude of the soft symbol estimate provides an estimated probability of the symbol, which is then used to decide whether the particular bit or symbol decision is reliable or not. However, it is impossible to extend the decision selection algorithm of [25] to the generic high-order QAM aided system. To the best of our knowledge, our method is the only available algorithm that can be used to select high-quality decisions for the generic QAM case. Moreover, even for BPSK signalling, the symbol probability estimate given in [25] itself may not always be reliable. This is indeed confirmed by the original simulation results for the BPSK case presented in [25], where it is seen that the performance loss is large at low SNRs in comparison to the perfect CSI performance bound. Indeed, the estimated probability of the $n$th bit or symbol is based on the single LLR $L_{p}^{I_{\text {in }}}(n)$ [25]. By contrast, our scheme utilizes all the $I_{\text {in }}$ LLRs provided by the inner iterative process to decide whether the $n$th detected bit or symbol is correct. We have also compared our algorithm to the decision selection algorithm of 
[25] designed for near-capacity BPSK MIMO systems, and the results obtained confirm that our scheme outperforms the scheme of [25].

Remark 3. The value of the block-of-bits selection threshold $T_{h}$ employed in step 2) for Criterion 1 should be carefully chosen. Too small a value may lead to an insufficient number of blocks selected for $\mathrm{CE}$ even after examining the entire sequence of $L_{F}$ bit decisions. By contrast, too large a value may result in the number of selected blocks reaching the limit value $M_{\text {sel }}$ after only examining a small initial portion of the $L_{F}$ bit decisions and the selected blocks may contain many "low confidence" decisions. Both of these two situations will result in a performance degradation. However, apart from these relatively extreme cases, our experience suggests that the performance of our semi-blind scheme is insensitive to the value of $T_{h}$. Specifically, there exists a relatively wide range of values for $T_{h}$, which allows our scheme to approach its optimal performance without increasing the number of turbo iterations. This range of optimal values for $T_{h}$ depends on both the modulation scheme and on the MIMO channel.

\section{Simulation Results And Discussions}

Example 1. A quasi-static Rayleigh fading MIMO system using $N_{T}=N_{R}=4$ and 16-QAM was simulated. An interleaver length of $L_{F}=16,000$ bits was used, yielding $M_{F}=1000$ for each 16-QAM symbol-vector frame. The binary generator polynomials of the half-rate RSC encoder were $G_{R S C}=[1,0,1]_{2}$ and $G_{R S C}^{r}=[1,1,1]_{2}$, while those of the URC encoder were $G_{U R C}=[1,0]_{2}$ and $G_{U R C}^{r}=[1,1]_{2}$. The transmitted signal power was normalised to unity, therefore the SNR was given as $\frac{1}{N_{0}}$. The number of initial training data blocks was chosen to be $M_{T}=6$, yielding a training overhead of $0.6 \%$, while the maximum number of selected blocks for our BBSB-SCE was limited to $M_{\mathrm{sel}}=100$. At the beginning of each frame, a new MIMO channel matrix $\boldsymbol{H}$ was generated by randomly drawing the channel taps according to $\mathcal{C N}(0,1)$, and $\boldsymbol{H}$ was kept constant in the frame duration. Two metrics were used to assess the achievable performance, namely the BER and the mean square error (MSE) of the CE. The CramérRao lower bound (CRLB) [26] is known to provide the best attainable performance for an unbiased estimator, and can be used for lower-bounding the MSE of a CE.

The BER performance of the proposed joint BBSB-SCE and three-stage turbo receiver is shown in Fig. 3, in comparison to that of the perfect CSI bound and those of the conventional semi-blind joint $\mathrm{CE}$ and three-stage turbo schemes utilising the entire detected data sequence for the soft and hard decision aided CE, respectively. Our semi-blind joint BBSB-SCE and three-stage turbo detector/decoder employed $I_{\text {in }}=3$ inner turbo iterations and $I_{\text {out }}=5$ outer turbo iterations, which were identical to those employed by the idealised three-stage turbo detector/decoder associated with the perfect CSI. It can be seen that the proposed semi-blind BBSB-SCE scheme is capable of attaining the near-capacity optimal ML performance associated with the perfect CSI, with the same "turbo-cliff" occurring before $\mathrm{SNR}=5 \mathrm{~dB}$. The conventional joint $\mathrm{CE}$ and three-stage turbo receiver combined with the soft-decision

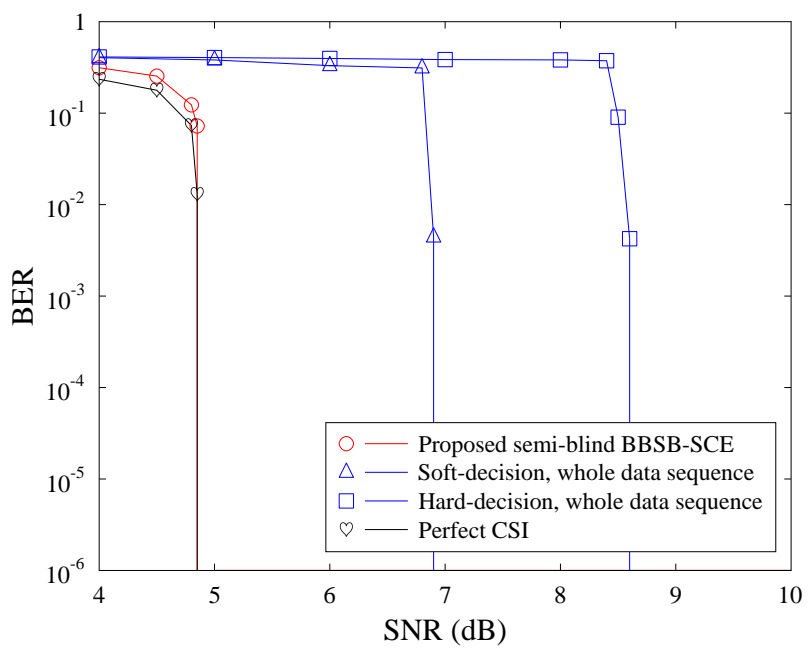

Fig. 3: BER performance comparison: a) perfect CSI case, b) proposed joint BBSB-SCE and three-stage turbo receiver with $T_{h}=1.0$, and c) conventional joint $\mathrm{CE}$ and three-stage turbo receivers employing the entire detected data sequence for the soft and hard decision aided channel estimators, respectively, for Example 1 of quasi-static MIMO system.

aided CE employing the entire detected data sequence cannot attain the perfect CSI performance bound, and there is a $2 \mathrm{~dB}$ gap between the BER turbo-cliffs of the two receivers. The conventional scheme employing the hard-decision aided CE based on the entire detected data sequence exhibits a further $1.5 \mathrm{~dB}$ degradation from its soft-decision assisted counterpart.

Fig. 4 illustrates the convergence behaviour of the proposed joint BBSB-SCE and three-stage turbo scheme. It can be seen that the BER gap between the proposed BBSB-SCE based scheme and the perfect CSI case reduces, as the number of outer iterations increases. Specifically, after the initial iteration there is a large BER gap, while during the third iteration the BER gap is reduced to around $1 \mathrm{~dB}$. Finally, at the fifth iteration there is no BER gap, indicating that the BBSB-SCE

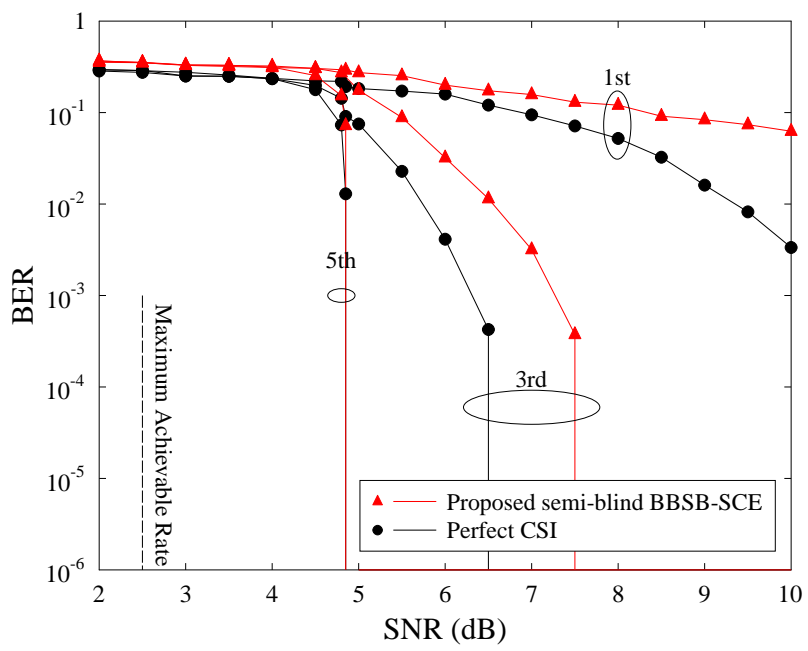

Fig. 4: Convergence performance of the proposed joint BBSB-SCE and three-stage turbo detector/decoder with $T_{h}=1.0$, in comparison to the perfect-CSI case, for Example 1 of quasi-static MIMO system. 


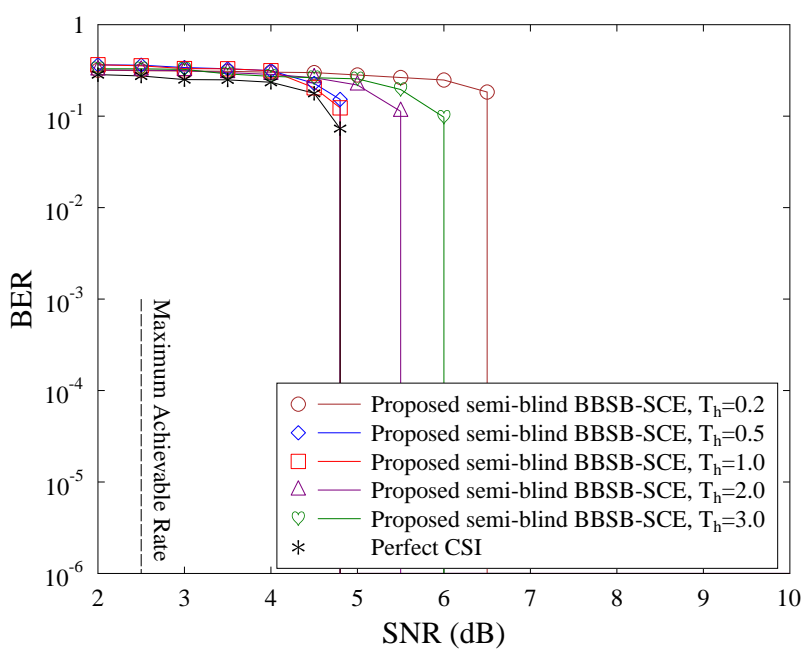

Fig. 5: Effects of the block-of-bits selection threshold $T_{h}$ on the achievable BER performance, for Example 1 of quasi-static MIMO system.

scheme has converged to the true MIMO CIR. This is very significant, since our semi-blind BBSB-SCE based scheme has as low a training overhead as $0.6 \%$ and yet it attains the optimal performance of the idealised three-stage turbo receiver associated with perfect CSI, while only imposing a complexity similar to the latter, as evidence by our complexity comparison given in (14). The effects of the selection threshold $T_{h}$ on the achievable performance of our proposed semi-blind scheme were investigated by varying the value of $T_{h}$ in the set $\{0.2,0.5,1.0,2.0,3.0\}$. The results obtained are shown in Fig. 5, where it is seen that $T_{h} \in[0.5,1.0]$ in this example allows our scheme to approach the perfect CSI performance bound. The MSE performance of the CE in our proposed scheme is compared to the CRLB associated with the optimal training sequence of length $M_{T}^{\mathrm{opt}}=100$ in Fig. 6, where it can be seen that the MSE of our DDCE approaches the

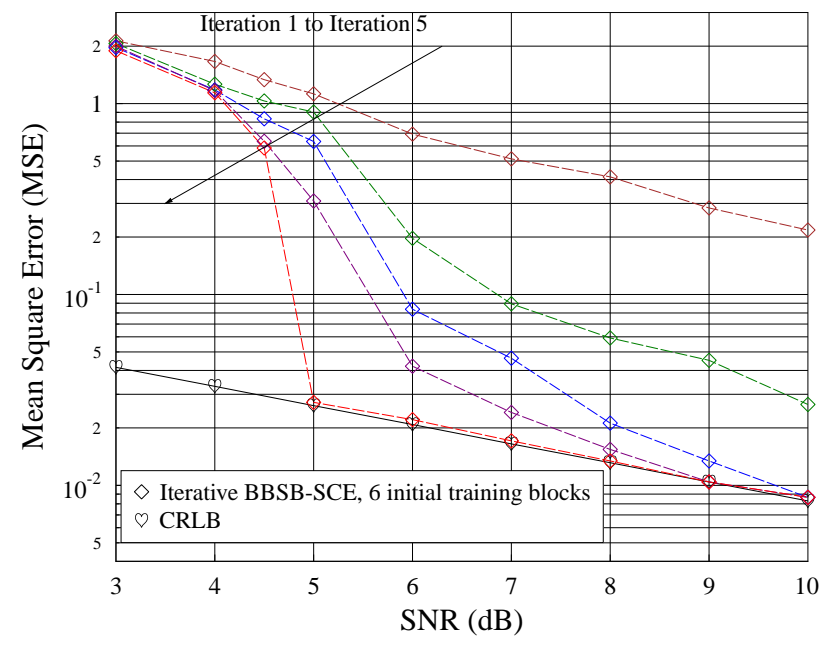

Fig. 6: MSE convergence performance of the CE in our proposed semi-blind joint BBSB-SCE and three-stage turbo detection/decoding scheme using a block-of-bits selection threshold of $T_{h}=1.0$, for Example 1 of quasi-static MIMO system.
CRLB, once the number of outer turbo iterations reaches $I_{\text {out }}=5$ for SNR $\geq 5.0 \mathrm{~dB}$. This corresponds to the BER cliff at $\mathrm{SNR} \approx 5.0 \mathrm{~dB}$ and $I_{\text {out }}=5$ shown in Fig. 3 .

Example 2. The system setup was identical to that of Example 1, except that the MIMO channels were time-varying. Specifically, $\boldsymbol{H}$ was faded at the symbol-rate during each frame according to the normalised Doppler frequency of $f_{d}$. Note that for the time-varying MIMO system, there exists a trade off between the time-varying channel's estimation (TVCE) performance and the turbo channel decoder's performance. To be more explicit, for turbo channel coding, a long interleaver length $L_{F}$ is preferred for the sake of achieving near-capacity performance [19]. However, a short frame length $M_{F}$, i.e. a short interleaver length $L_{F}$ is preferred for the sake of achieving a good TVCE performance. In our simulations, we varied $f_{d}$ and investigated different interleaver lengths ${ }^{4}$.

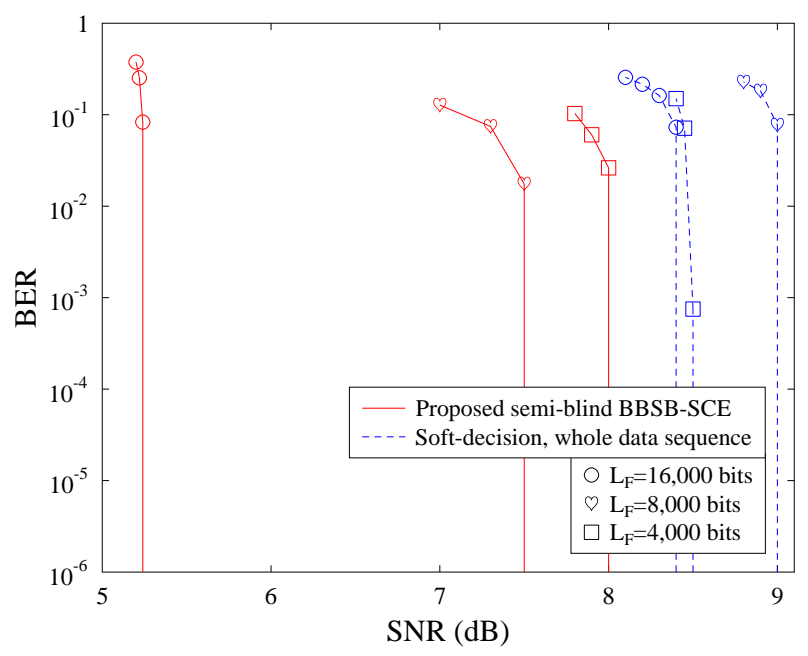

Fig. 7: BER performance comparison: a) proposed joint BBSB-SCE and three-stage turbo receiver with $T_{h}=1.0$, and b) conventional joint $\mathrm{CE}$ and three-stage turbo receiver employing the entire detected data sequence for the soft decision aided channel estimator, for Example 2 of time-varying MIMO system with the normalised Doppler frequency $f_{d}=10^{-5}$ as well as the interleaver lengths of $L_{F}=16,000$ bits, 8,000 bits and 4,000 bits, respectively.

Fig. 7 shows the results obtained for the case of the normalised Doppler frequency $f_{d}=10^{-5}$ with $L_{F}=$ 16,000 bits, 8,000 bits and 4,000 bits, respectively, where it can be seen that for each given interleaver length $L_{F}$ the proposed semi-blind joint BBSB-SCE relying on three-stage turbo receiver outperforms the conventional semi-blind joint $\mathrm{CE}$ and three-stage turbo scheme utilising the entire detected data sequence. Specifically, our scheme achieves SNR gains of $3.1 \mathrm{~dB}, 1.5 \mathrm{~dB}$ and $0.5 \mathrm{~dB}$ over the conventional one for $L_{F}=16,000$ bits, $L_{F}=8,000$ bits and $L_{F}=4,000$ bits, respectively. As expected, our proposed semi-blind BBSBSCE scheme achieves its best BER performance for the long interleaver length of $L_{F}=16,000$ bits. This is because the normalized Doppler frequency of $f_{d}=10^{-5}$ represents a relatively slowly fluctuating channel. Hence the achievable

\footnotetext{
${ }^{4}$ In practice, Doppler spread may be estimated using the schemes proposed in [27]-[29].
} 
system performance is dominated by the performance of iterative channel decoder which favours a high $L_{F}$ value. Furthermore, as the interleaver length of our scheme reduces, the number of potential high-quality candidates may also be reduced, which may hence contribute to the degradation of the system's performance.

A similar conclusion may be drawn for the conventional semi-blind CE scheme from Fig. 7, where the performance of the conventional scheme is degraded by about $0.6 \mathrm{~dB}$, when the interleaver length is reduced from 16,000 bits to 8,000 bits. However, unlike for our proposed semi-blind BBSB-SCE scheme, in this particular case, the performance of the conventional semi-blind CE scheme recorded for $L_{F}=4,000$ bits is slightly better than that of the $L_{F}=8,000$-bit scenario.

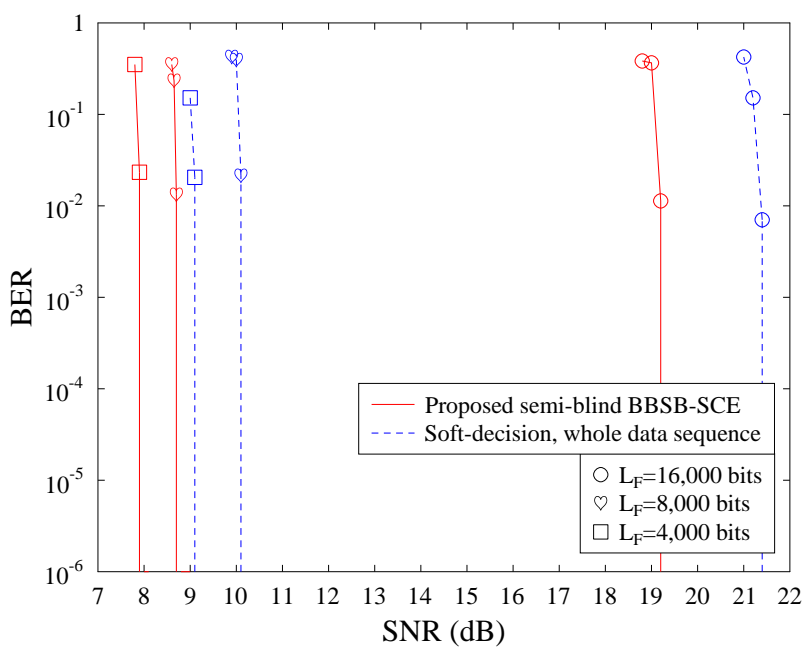

Fig. 8: BER performance comparison: a) proposed joint BBSB-SCE and three-stage turbo receiver with $T_{h}=1.0$, and b) conventional joint $\mathrm{CE}$ and three-stage turbo receiver employing the entire detected data sequence for the soft decision aided channel estimator, for Example 2 of time-varying MIMO system with the normalised Doppler frequency $f_{d}=10^{-4}$ as well as the interleaver lengths of $L_{F}=16,000$ bits, 8,000 bits and 4,000 bits, respectively.

Fig. 8 compares the achievable BER performance of our proposed scheme to that of the conventional scheme for the case of the normalised Doppler frequency $f_{d}=10^{-4}$ with $L_{F}=16,000$ bits, 8,000 bits and 4,000 bits, respectively. As seen from Fig. 8, the best performance is achieved with the interleaver length of $L_{F}=4,000$ bits, while the worst performance is obtained for the interleaver length of $L_{F}=$ 16,000 bits for both our proposed scheme and the conventional one. At the normalised Doppler frequency of $f_{d}=10^{-4}$ the MIMO system's overall performance is dominated by the TVCE performance, which favours a short interleaver. Evidently, there exists a trade off between the turbo channel coding performance and the TVCE performance in choosing the best interleaver length. Observe furthermore in Fig. 8 that for a given interleaver length $L_{F}$, our proposed scheme always outperforms the conventional one.

When considering an even higher normalised Doppler frequency of $f_{d}=5 \times 10^{-4}$, both our proposed semi-blind joint BBSB-SCE relying on three-stage turbo receiver as well as the

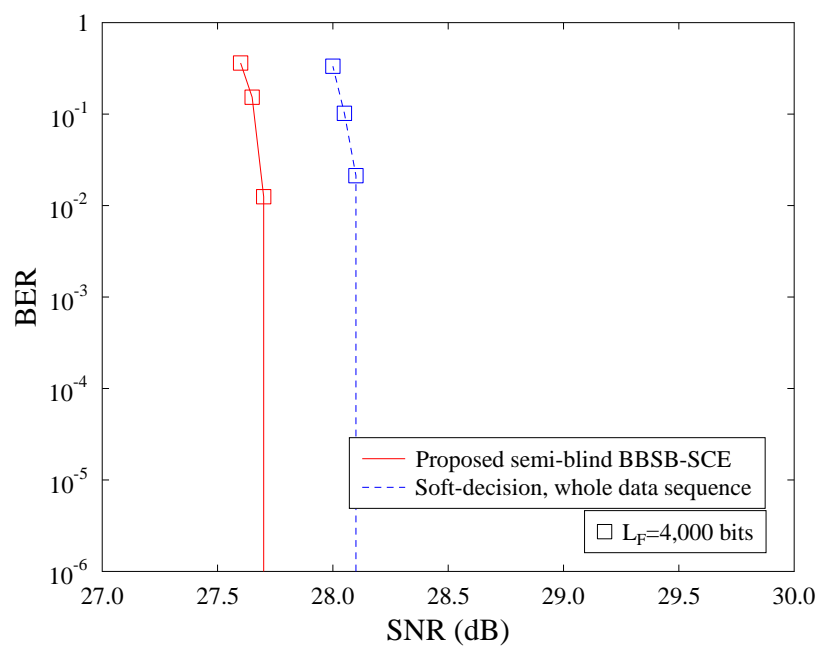

Fig. 9: BER performance comparison: a) proposed joint BBSB-SCE and three-stage turbo receiver with $T_{h}=1.0$, and b) conventional joint $\mathrm{CE}$ and three-stage turbo receiver employing the entire detected data sequence for the soft decision aided channel estimator, for Example 2 of time-varying MIMO system with the normalised Doppler frequency $f_{d}=5 \times 10^{-4}$ and the interleaver length of $L_{F}=4,000$ bits.

conventional semi-blind joint $\mathrm{CE}$ and three-stage turbo scheme utilising the entire detected data sequence for the soft-decision aided CE cannot converge (there exists no open tunnel between the EXIT curves of the inner and outer decoders) for the interleaver lengths of $L_{F}=16,000$ bits and $L_{F}=8,000$ bits associated with an SNR as high as $30 \mathrm{~dB}$. Evidently, using $L_{F}=16,000$ bits or $L_{F}=8,000$ bits is excessively long, which degrades the TVCE performance to an unacceptable level. However, when the interleaver length was reduced to $L_{F}=4,000$, both schemes become capable of achieving convergence, as seen from Fig. 9. Observe from Fig. 9 that the proposed semi-blind BBSB-SCE scheme outperforms the

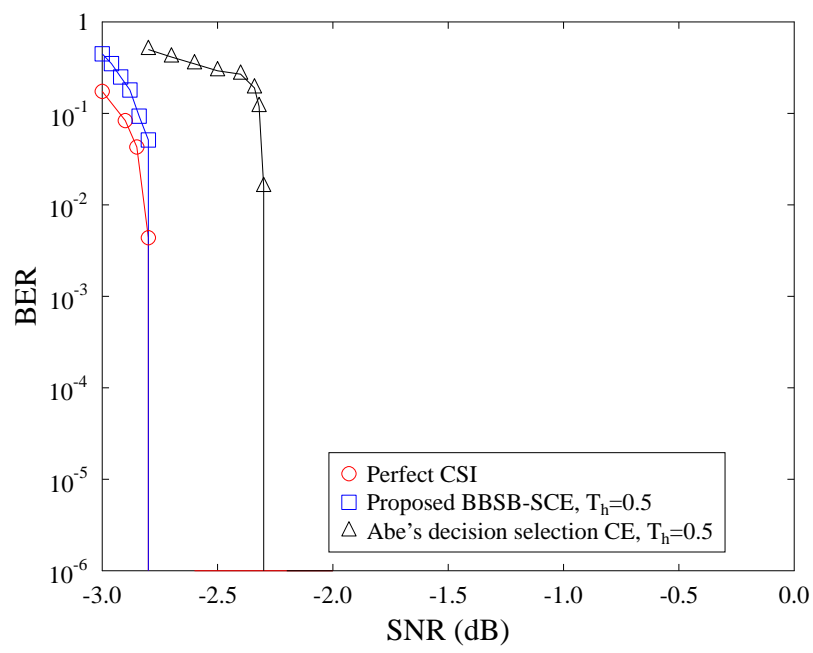

Fig. 10: BER performance comparison: a) perfect CSI case, b) proposed joint BBSB-SCE and three-stage turbo receiver with $T_{h}=0.5$, and c) Abe and Matsumoto's BPSK decision selection scheme based soft CE [25], for Example 3 of quasi-static BPSK MIMO system with $N_{T}=N_{R}=2$. 
conventional semi-blind $\mathrm{CE}$ scheme by about $0.4 \mathrm{~dB}$.

Example 3. We also considered a quasi-static Rayleigh fading BPSK MIMO system associated with $N_{T}=N_{R}=2$ and set the initial training block length to $M_{T}=6$. The other system settings were the same as in Example 1. The simulation results shown in Fig. 10 compare our proposed BBSB-SCE scheme to the decision selection scheme of Abe and Matsumoto based on soft CE [25], using again the perfect CSI performance bound as the benchmark. It can be explicitly seen in Fig. 10 that as expected, our BBSB-SCE scheme approaches the optimal performance bound associated with the perfect CSI and it slightly outperforms the scheme proposed by Abe and Matsumoto by about $0.5 \mathrm{~dB}$.

\section{Conclusions}

We have proposed a novel semi-blind joint BBSB-SCE and three-stage turbo detection/decoding scheme for nearcapacity MIMO systems. Unlike all the existing methods, our scheme does not require an extra iterative loop between the channel estimator and the turbo detector/decoder, since our BBSB-SCE is naturally embedded into the original three-stage demapping/decoding turbo loop. This novel arrangement enables us to substantially reduce the computational complexity. Most significantly, our BBSB-SCE scheme only selects highconfidence decisions for our soft-DDCE. This not only ensures that the complexity of our channel estimator is several orders of magnitude lower than that of the existing methods, but also enables our scheme to attain the optimal ML performance of the idealised three-stage turbo receiver furnished with perfect CSI, using the same number of turbo iterations as the latter.

\section{REFERENCES}

[1] M. Loncar, R. Muller, J. Wehinger, and T. Abe, "Iterative joint detection, decoding, and CE for dual antenna arrays in frequency selective fading," in Proc. 5th Int. Symp. Wireless Personal Multimedia Communications (Honolulu, Hawaii), Oct. 27-30, 2002, pp. 125-129.

[2] M. Loncar, R. Muller, J. Wehinger, C. F. Mecklenbrauker, and T. Abe, "Iterative channel estimation and data detection in frequency-selective fading MIMO channels," European Trans. Telecommunications, vol. 15, no. 5, pp. 459-470, Sept./Oct. 2004.

[3] H. Li, S. M. Betz, and H. V. Poor, "Performance analysis of iterative channel estimation and multiuser detection in multipath DS-CDMA channels," IEEE Trans. Signal Processing, vol. 55, no. 5, pp. 19811993, May 2007.

[4] M. Jiang, J. Akhtman, and L. Hanzo, "Iterative joint CE and multiuser detection for multiple-antenna aided OFDM systems," IEEE Trans. Wireless Communications, vol. 6, no. 8, pp. 2904-2914, Aug. 2007.

[5] M. Qaisrani and S. Lambotharan, "An iterative (turbo) CE and symbol detection technique for doubly selective channels," in Proc. VTC2007Spring (Dublin, Ireland), April. 22-25, 2007, pp. 2253-2256.

[6] P. S. Rossi and R. R. Müller, "Joint twofold-iterative channel estimation and multiuser detection for MIMO-OFDM systems," IEEE Trans. Wireless Communications, vol. 7, no. 11, pp. 4719-4729, Nov. 2008.

[7] J. Ylioinas and M. Juntti, "Iterative joint detection, decoding, and channel estimation in turbo-coded MIMO-OFDM," IEEE Trans. Vehicular Technology, vol. 58, no. 4, pp. 1784-1796, May 2009.

[8] J. Zhang, S. Chen, X. Mu, and L. Hanzo, "Joint CE and multi-user detection for SDMA/OFDM based on dual repeated weighted boosting search," IEEE Trans. Vehicular Technology, vol. 60, no. 7, pp. 32653275, 2011.

[9] J. Zhang, S. Chen, X. Mu, and L. Hanzo, "Turbo multi-user detection for OFDM/SDMA systems relying on differential evolution aided iterative channel estimation," IEEE Trans. Communications, vol. 60, no. 6, pp. 1621-1633, June 2012.
[10] M. Sandell, C. Luschi, P. Strauch, and R. Yan, "Iterative CE using soft decision feedback," in Proc. Globecom'1998 (Sydney, Australia), Nov. 8-12, 1998, pp. 3728-3733.

[11] S. Song, A. C. Singer, and K. Sung, "Soft input CE for turbo equalization," IEEE Trans. Signal Processing, vol. 52, no. 10, pp. 2885-2894, Oct. 2004.

[12] B. Hu, A. Kocian, R. Piton, A. Hviid, B. H. Fleury, and L. K. Rasmussen, "Iterative joint $\mathrm{CE}$ and successive interference cancellation using a SISO-SAGE algorithm for coded CDMA," in Proc. 38th Asilomar Conf. Signals, Systems and Computers (Pacific Grove, CA), Nov. 7-10, 2004, pp. 622-626.

[13] M. Sellathurai and S. Haykin, "Turbo-BLAST for wireless communications: Theory and experiments," IEEE Trans. Signal Processing, vol. 50, no. 10, pp. 2538-2546, Oct. 2002.

[14] M. Nicoli, S. Ferrar, and U. Spagnolini, "Soft-iterative channel estimation: methods and performance analysis," IEEE Trans. Signal Processing, vol. 55, no. 6, pp. 2993-3006, June 2007.

[15] Y. Wu, X. Zhu, and A. K. Nandi, "Soft-input turbo CE for singlecarrier multiple-input-multiple-output systems," IEEE Trans. Vehicular Technology, vol. 58, no. 7, pp. 3867-3873, Sept. 2009.

[16] W. Haselmayr, D. Schellander, and A. Springer, "Iterative CE and turbo equalization for time-varying channels in a coded OFDM-LTE system for 16-QAM and 64-QAM," in Proc. 21st IEEE Int. Symp. Personal Indoor and Mobile Radio Communications (Istanbul, Turkey), Sept. 2629, 2010, pp. 614-618.

[17] I. Land, P. A. Hoeher, and S. Gligorević, "Computation of symbol-wise mutual information in transmission systems with $\log$ APP decoders and application to EXIT charts," in Proc. 5th Int. ITG Conf. Source and Channel Coding (SCC) (Erlangen, Germany), Jan. 2004, pp. 195-202.

[18] J. Wang, S. X. Ng, A. Wolfgang, L. L. Yang, S. Chen, and L. Hanzo, "Near-capacity three-stage MMSE turbo equalization using irregular convolutional codes," in: Proc. Turbo-Coding-2006 (Munich, Germany), April 3-7, 2006, 6 pages.

[19] L. Hanzo, O. R. Alamri, M. El-Hajjar, and N. Wu, Near-Capacity Multi-Functional MIMO Systems: Sphere-Packing, Iterative Detection and Cooperation. John Wiley \& Sons: Chichester, UK, 2009.

[20] R. G. Maunder and L. Hanzo, "Iterative decoding convergence and termination of serially concatenated codes," IEEE Trans. Vehicular Technology, vol. 90, no. 1, pp. 216-224, Jan. 2010.

[21] L. Hanzo, S.X. Ng, T. Keller and W. Webb, Quadrature Amplitude Modulation: From Basics to Adaptive Trellis-Coded, Turbo-Equalised and Space-Time Coded OFDM, CDMA and MC-CDMA Systems, 2nd edition. Chichester, UK: John Wiley, 2004.

[22] C. Xu, S. Sugiura, S. X. Ng, and L. Hanzo, "Spatial modulation and space-time shift keying: Optimal performance at a reduced detection complexity," IEEE Trans. Communications, vol. 61, no. 1, pp. 206-216, Jan. 2013.

[23] Z. Guo and P. Nilsson, "Algorithm and implementation of the Kbest sphere decoding for MIMO detection," IEEE J. Selected Areas in Communications, vol. 24, no. 3, pp. 491-503, March 2006.

[24] L. Wang, L. Xu, S. Chen, and L. Hanzo, "Apriori-LLR-threshold-assisted K-best sphere detection for MIMO channels," in Proc. VTC2008-Spring (Singapore), May 11-14, 2008, pp. 867-871.

[25] T. Abe and T. Matsumoto, "Space-time turbo equalization in frequencyselective MIMO channels," IEEE Trans. Vehicular Technology, vol. 52, no. 3, pp. 469-475, May 2003.

[26] S. M. Kay, Fundamentals of Statistical Signal Processing: Estimation Theory. Englewood Cliffs, NJ: Prentice-Hall, 1993.

[27] J. Hua, L. Meng, X. Xu, D. Wang, and X. You, "Novel scheme for joint estimation of SNR, Doppler, and carrier frequency offset in doubleselective wireless channels," IEEE Trans. Vehicular Technology, vol. 58, no. 3, pp. 1204-1217, March 2009.

[28] J. Hua, L. Meng, G. Li, D. Wang, B. Sheng, and X. You, "Joint estimation of channel parameters for very low signal-to-noise ratio environment in mobile radio propagations," Radio Science, vol. 45, no. 4, Aug. 2010.

[29] A. Dogandzic and B. Zhang, "Estimating Jakes' Doppler power spectrum parameters using the whittle approximation," IEEE Trans. Signal Processing, vol. 53, no. 3, pp. 987-1005, March 2005. 